\title{
Heating characteristics and atomic emission waveforms for glow discharge ion bombardment furnaces
}

\author{
Suzanne Tanguay and Richard Sacks* \\ Department of Chemistry, University of Michigan, Ann Arbor, MI 48109, U.S.A.
}

(Received 15 May 1990; accepted 20 August 1990)

\begin{abstract}
Ion bombardment of a graphite cathode in a glow discharge plasma is used to heat the cathode to temperatures suitable for the vaporization of solution residues from the cathode surface. Two low-mass cathode configurations are considered. The use of a cylindrical post cathode results in very efficient heating with peak temperatures of about $2500^{\circ} \mathrm{C}$ for a $250 \mathrm{~mA}$ discharge in Ar at 4.0 torr $(532 \mathrm{~Pa})$. The use of a hollow cathode results in longer sample vapor residence time, but the greater surface area and lower plasma voltage result in less efficient heating, and peak temperatures are about $2100^{\circ} \mathrm{C}$. Optical pyrometer data are presented showing the effects of cathode configuration, plasma current and argon pressure on furnace temperature. The effect of an axial magnetic field on cathode heating also is considered. The role of thermionic electron emission in limiting peak furnace temperature and in regulating the temperature is discussed.
\end{abstract}

\section{INTRODUCTION}

THE COMBINATION of electrothermal (furnace) vaporization and electron-impact excitation in a low-pressure glow discharge has been shown to have attractive features for the determination of metallic elements in solution residue samples. Furnace atomic nonthermal excitation spectroscopy (FANES) uses a graphite tube furnace as the cathode element in the glow-discharge plasma which is usually operated in $\mathrm{Ar}$ at a pressure of a few torr [1-5]. The furnace tube is heated resistively by a low-voltage, $2.5-\mathrm{kW}$ power supply. A separate, higher voltage power supply is used to maintain the hollow-cathode glow discharge in the furnace tube. Plasma current and cathode current density typically have values of about $30 \mathrm{~mA}$ and $4 \mathrm{~mA} / \mathrm{cm}^{2}$, respectively. Detection limits typically are comparable to furnace atomic absorption values.

Recently, Harnly et al. [6,7] described a similar technique using a hollow anode configuration (HA-FANES). Here, the graphite tube furnace is used as the anode in the glow discharge, and a coaxial carbon rod serves as the cathode. This configuration has the advantage of greater plasma stability and eliminates the need for electrical shielding in order to keep the plasma inside of the cylindrical hollow furnace electrode.

TANGUAY and SACKS [8] described a unique glow-discharge furnace system in which a cylindrical post cathode, which serves as the furnace, is heated by positive ion bombardment from the glow discharge. Only a single 250-mA power supply is required. Heating by positive ion bombardment is quite efficient, and in $\mathrm{Ar}$ in the pressure range 1-4 torr (133-532 $\mathrm{Pa}$ ), satisfactory sample atomization temperatures were reached in a few seconds. A magnetic field of a few hundred Gauss applied parallel to the cathode axis had a dramatic effect on lowering the plasma voltage and increasing emission signals for solution residue samples atomized from the cathode surface and excited in the glow-discharge plasma. However, the cylindrical post design results in very short sample vapor residence times.

In the present study, a graphite ion bombardment furnace using a hollow cathode configuration is evaluated and compared to the cylindrical post configuration. Direct optical pyrometer measurements of furnace surface temperature are reported for both

\footnotetext{
* Author to whom correspondence should be addressed.
} 
cathode configurations. The effect of an external magnetic field on furnace temperature is considered. The role of thermionically emitted electrons from the hot graphite surface in establishing an upper limit on ion bombardment heating also is considered.

\section{EXPERIMENTAL}

\subsection{Furnace design}

The cylindrical post geometry evaluated in this study was described previously [8]. It consists of a 1.6-mm diameter, 12-mm long cylinder machined on the end of a 3.05-mm diameter graphite rod. A diagram of this electrode geometry is shown in Fig. 1(a). Note that a ceramic insulator (I) covers the other portion of the graphite cylinder (G) thus restricting plasma formation to the 12-mm long post and the exposed end of the rod. The surface area exposed to the plasma is about $0.60 \mathrm{~cm}^{2}$, not including the flat surfaces normal to the magnetic field. The mass of the post is about $0.054 \mathrm{~g}$.

The hollow cathode geometry, shown at the top of Fig. 1(b), was machined from the same $3.05-\mathrm{mm}$ diameter graphite stock as the cylindrical post geometry. The cathode cavity is $2.36 \mathrm{~mm}$ in diameter and $12.7 \mathrm{~mm}$ deep. Note that the cavity wall thickness is only $0.34 \mathrm{~mm}$. The cylinder wall has a mass of about $0.33 \mathrm{~g}$ and a total surface area of about $2.20 \mathrm{~cm}^{2}$. This includes both the inner and the outer surfaces of the wall. A $0.8-\mathrm{mm}$ diameter hole is located $6.4 \mathrm{~mm}$ from the open end of the cavity. This hole is used for the introduction of a micro solution sample.

\subsection{Glow discharge design}

The cathode assembly is located along the axis of a cylindrical polycarbonate plastic chamber equipped with a quartz observation window, a gas-tight sample introduction port, and connections for the vacuum pump, gas inlet and thermocouple pressure gauge. The cathode assembly is

(a)

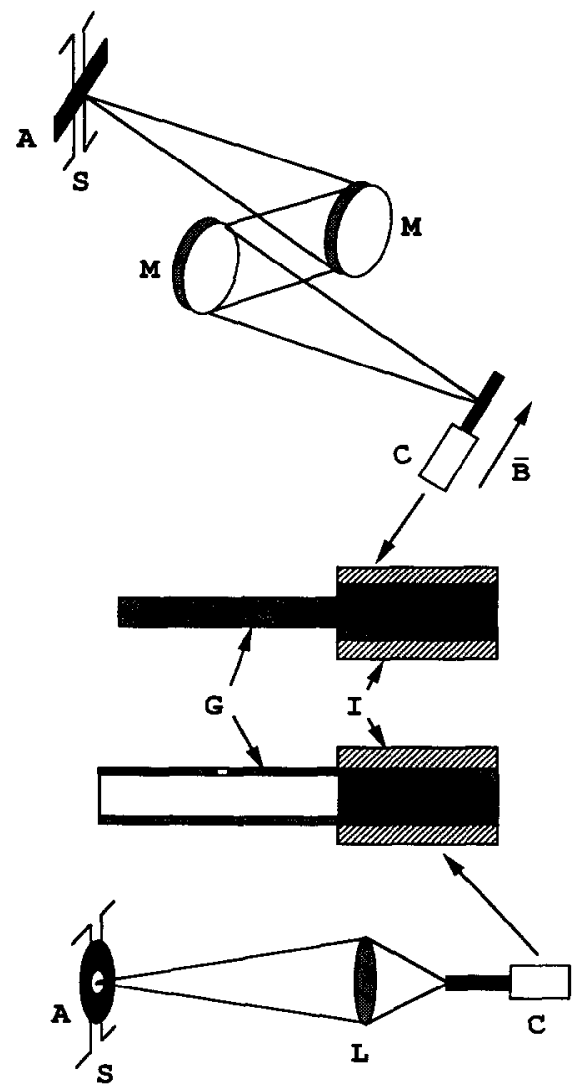

Fig. 1. Cathode configurations and optical systems for ton-bombardment furnaces. (a) Cylindrical post furnace geometry, (b) hollow cathode furnace geometry. G, graphite furnace; I, ceramic insulator; $\mathrm{C}$, cathode assembly; B. magnetic field vector; $\mathrm{M}$, mirror; L, lens; S, spectrometer slit; A, field-stop aperture. 
mounted on a water-cooled copper cylinder which is attached to the Bakelite end plate of the chamber. The anode consists of a $5.0-\mathrm{cm}$ o.d., $4.2-\mathrm{cm}$ i.d., $2.0-\mathrm{mm}$ thick copper ring which is coaxial with the cathode.

For some experiments, the chamber was placed in the pole gap of a water-cooled electromagnet (CENCO Model 79637-077). This magnet has an adjustable pole gap, and in order to accommodate the plasma chamber, the gap was opened to $10 \mathrm{~cm}$. Under these conditions, the peak field strength was about $1.24 \mathrm{kG}$. For all experiments, the cathode axis was parallel to the magnetic field.

The lamp was powered by a Glassman Model PS/PG-003N-250 power supply which has constant current operation in the range $0-250 \mathrm{~mA}$ with output voltage up to $3.0 \mathrm{kV}$. The higher voltages are needed with the cylindrical post cathode configuration.

Argon plasma gas was passed through filters for oxygen, water vapor and hydrocarbons and finally through a liquid-nitrogen trap. Gas flow and pressure were adjusted by means of a Brooks Model 8744 flow control valve. Pressure was measured with a Hastings-Raydist Model $\mathrm{CVH} 4$ thermocouple gauge equipped with a type DV-24 gauge tube. The chamber was pumped by a two-stage mechanical pump. A liquid-nitrogen trap was located between the pump and the chamber.

\subsection{Optical and electrical monitoring}

Cathode temperature measurements were made using a Leeds and Northrup Model 8622-C hand-held optical pyrometer. The pyrometer uses a hot filament with an adjustable current source to compare the surface color temperature of the target. The lower temperature limit of the instrument is $1100^{\circ} \mathrm{C}$, and the accuracy is about $1 \%$ of the measured temperature.

Radiation was monitored using a $0.75-\mathrm{m}$ Czerny-Turner spectrometer equipped with a 1200 line $/ \mathrm{mm}$ grating blazed for $300 \mathrm{~nm}$ in the first order. Slit widths were $100 \mu \mathrm{m}$, resulting in a spectral bandwidth of $0.11 \mathrm{~nm}$. Photoelectric radiation intensity data were obtained with a $1 \mathrm{P} 28$ photomultiplier tube biased at $900 \mathrm{~V}$. Resistive load was adjusted as needed. For most measurements, the circuit time constant was $30 \mathrm{~ms}$.

Figure 1(a) shows the optical system used with the cylindrical-post cathode furnace. The axis of cathode $C$ was oriented in the horizontal plane and normal to the optical axis. The coaxial magnetic field is represented by vector $B$. A pair of $5.0-\mathrm{cm}$ dia, $50-\mathrm{cm}$ focal length spherical mirrors $M$ was used in an over-and-under configuration to image the cathode onto the plane of the spectrometer entrance slit $\mathrm{S}$. The region of the plasma located about $6 \mathrm{~mm}$ from the end of the cathode was imaged onto the entrance slit with unit magnification. A solid mask A, $1.6 \mathrm{~mm}$ in height, was placed horizontally in front of the entrance slit in order to block the incandescent radiation from the hot cathode. This configuration allows for symmetric observation above and below the cathode.

Figure 1(b) shows the optical system used with the hollow cathode furnace. The cathode axis was oriented in the horizontal plane and parallel to the optical axis. This results in end-on observation, and a $5.0-\mathrm{cm} \mathrm{dia.,} 15-\mathrm{cm}$ focal length lens $\mathrm{L}$ was used to image radiation from the circular end of the cathode onto the plane of the entrance slit $\mathrm{S}$ with a magnification of 3.0. A field-stop iris $\mathrm{A}$ was placed directly in front of the entrance slit in order to block incandescent radiation from the cathode wall.

Photoelectric data were collected at a sampling rate of $100 \mathrm{~Hz}$ using a Nicolet Model 2090 digital storage oscilloscope or an IBM-PC Model 5150 computer equipped with an 8-bit A/D board (Metrabyte Corp. DAS-8). Plasma voltage was measured at a voltage monitoring terminal on the power supply. Magnetic field strength was measured with a Hall-effect Gaussmeter (A\&I Industries Model 100).

\section{Results ANd Discussion}

\subsection{Furnace heating processes}

For the conditions used here, furnace heating is almost entirely by interaction with the plasma. Ohmic dissipation in the graphite is negligible. Direct energy transfer during positive ion bombardment and convective heating from the hot plasma gas in the sheath surrounding the cathode are the principal heating processes. At low temperatures, nearly all of the primary electrons (electrons emitted by the cathode) needed to sustain the plasma are generated from positive ion bombardment of the cathode surface. At higher temperatures, thermionic electron emission will become significant. 
Thermionic electron current density $j$ is related to the surface temperature $T$ and the surface work function $\phi[9]$.

$$
j=A T^{2} \exp [-e \phi / k T]
$$

where $A$ is a constant for a given surface. For carbon, the values of $4.4 \mathrm{eV}$ for $\phi$ and 48 for $A$ were used [10], and the calculated thermionic temperature vs current plots shown in Fig. 2 were obtained for surface areas of $0.63 \mathrm{~cm}^{2}(\mathrm{~A}), 1.38 \mathrm{~cm}^{2}$ (B) and $2.16 \mathrm{~cm}^{2}(\mathrm{C})$. These values are comparable with values for the cylindrical post and the hollow cathode configurations used here.

Note that the plasma currents used with the furnaces described in this report are in the range $150-250 \mathrm{~mA}$. Thus, for furnace temperatures greater than about $2000^{\circ} \mathrm{C}$, thermionic emission can supply a large fraction of the total plasma current. Since the emission of these electrons does not depend on positive ion bombardment, the plasma voltage decreases significantly at these furnace temperatures, and the plasma assumes more arc-like characteristics [8]. Note that the curves are quite flat at the higher temperatures, and a small change in furnace temperature results in a relatively large change in thermionic current. This feature may provide some temperature regulation, and may make it difficult to heat the furnace to significantly higher temperatures using only positive ion bombardment from the plasma.

\subsection{Cylindrical post cathode}

The cylindrical-post cathode furnace shows dramatic variations in plasma voltage during a heating cycle [8]. When the discharge is ignited, the voltage first increases rapidly with increasing temperature. This may be due, in part, to gas rarefaction which occurs in the vicinity of the cathode as it heats $[11,12]$. After reaching a peak value at relatively low temperature, the voltage falls with continued heating. This is probably the result of the increasing contribution of thermionic electrons to the total plasma current. The peak plasma voltage is strongly dependent on Ar pressure and magnetic field strength. For the relatively high cathode current densities needed for rapid and efficient furnace heating, the peak plasma voltage may exceed $3 \mathrm{kV}$.

If a magnetic field is applied parallel to the furnace axis, the rate of radial electron diffusion is reduced [13], and the plasma voltage is reduced. Figure 3 shows plots of the cylindrical-post furnace surface temperature from optical pyrometer measurements vs the plasma current. Data are shown for Ar pressures of 1.0, 2.0 and 4.0 torr. Magnetic fields of $0.64,0.92$ and $1.24 \mathrm{kG}$ were used. These temperature values were obtained under steady-state conditions after the plasma voltage had stabilized. Note that the emissivity of the graphite surface is less than 1.0, and thus the optical pyrometer data may underestimate the surface temperature.

For magnetic field strengths less than about $0.64 \mathrm{kG}$, the plasma voltage for the

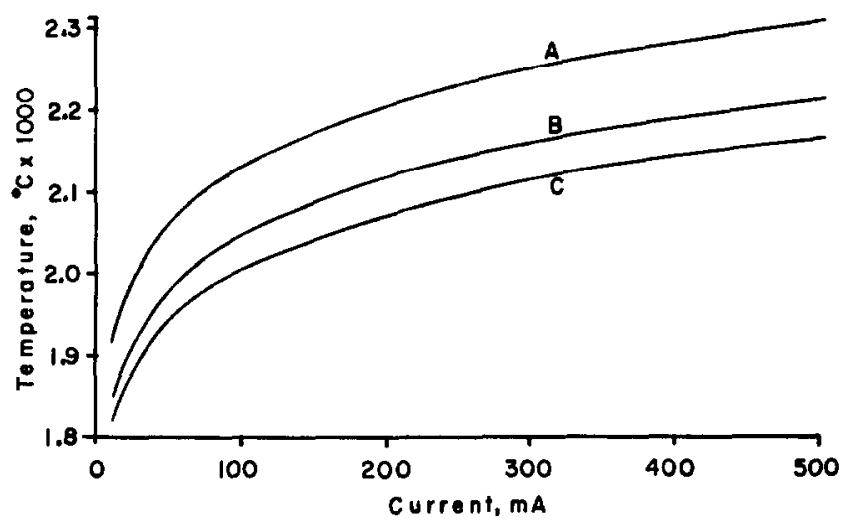

Fig. 2. Plots of surface temperatures vs thermionic current for carbon surfaces with areas of $0.60 \mathrm{~cm}^{2}$ (A), $1.38 \mathrm{~cm}^{2}$ (B) and $2.16 \mathrm{~cm}^{2}$ (C). 
(a)

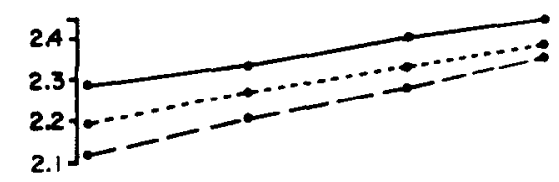

(b)

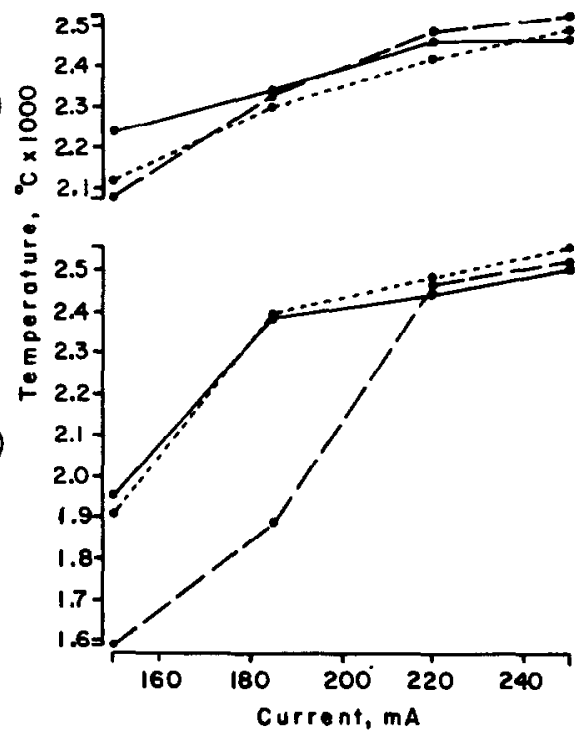

Fig. 3. Plots of furnace temperature vs plasma current for the cylindrical post furnace with $\mathrm{Ar}$ pressures of 1.0 torr (a), 2.0 torr (b) and 4.0 torr (c). For the solid-line plots, a $0.64-\mathrm{kG}$ magnetic field was applied parallel to the furnace axis. The field strength was 0.92 and $1.24 \mathrm{kG}$ for the dotted-line and broken-line plots, respectively.

1.0-torr case exceeded the $3.0 \mathrm{kV}$ available from the power supply, and constant current operation could not be achieved at the highest current values used here. As the magnetic field strength is increased, the plasma voltage drops significantly. Peak voltage values observed at 1.0 torr with a plasma current of $200 \mathrm{~mA}$ were $2.6,1.6$ and $1.1 \mathrm{kV}$ for magnetic field strengths of $0.64,0.92$ and $1.24 \mathrm{kG}$, respectively.

At a pressure of 1.0 torr, the furnace heats very efficiently, and the steady-state temperature values shown in Fig. 3(a) were achieved in about $2 \mathrm{~s}$. For all magnetic field strengths used here, the furnace temperature increases rather slowly with increasing plasma current. An increase in magnetic field strength results in a decrease in temperature and in a decrease in the steady-state plasma voltage. With a plasma current of $200 \mathrm{~mA}$, the steady-state voltage has values of about $1.0,0.75$ and $0.65 \mathrm{kV}$ for field strengths of $0.64,0.93$ and $1.24 \mathrm{kG}$, respectively. Thus, the lower temperatures observed with the higher magnetic field strengths, in part, can be attributed to the reduced power dissipation in both the plasma and the furnace.

When the Ar pressure is increased to 2.0 torr, the steady-state furnace temperature increases more rapidly with increasing plasma current, and at the higher current values, temperatures are greater than for the 1.0 torr case. This trend is more pronounced at the higher magnetic field strengths. Note that at a pressure of 2.0 torr, the steadystate plasma voltage is about $0.5 \mathrm{kV}$ for a current of $200 \mathrm{~mA}$. This value is nearly independent of magnetic field strength for the values used here.

When the pressure is increased to 4.0 torr, the furnace temperature is more strongly dependent on the plasma current for the range of values used here. The steady-state temperatures achieved with the 0.64 and $0.92 \mathrm{kG}$ magnetic fields are not significantly different. However, with the $1.24-\mathrm{kG}$ field, the temperatures achieved are much lower for the lower current values. At a current of $200 \mathrm{~mA}$, the steady-state plasma voltage is about $0.4 \mathrm{kV}$ and is nearly independent of the magnetic field strength. For the higher current values used here, the furnace temperature is in the range $2500-2600^{\circ} \mathrm{C}$ for all three magnetic field strengths. 
Figure 4 shows the effects of Ar pressure and magnetic field strength on the emission intensity vs time profiles for the $\mathrm{Mn}$ 403.1-nm neutral-atom line (a) and continuum background at the same wavelength (b). Data were collected at Ar pressures of 1.0 and 4.0 torr and with magnet field strengths of 0.64 and $1.24 \mathrm{kG}$. All data were obtained with $200-\mathrm{mA}$ heating pulses. In each case, 5.0-ng Mn samples were deposited as $5.0 \mu \mathrm{l}$ of $1.0 \mu \mathrm{g} / \mathrm{ml} \mathrm{Mn}\left(\mathrm{NO}_{3}\right)_{2}$ solution. A distilled-water blank was used for the background profiles.

Note that the greatest line intensity and line-to-background intensity ratio values are obtained for the 4.0-torr case with the stronger magnetic field For the 1.24-kG case, the peak furnace temperatures are nearly the same at the two pressures. However, the initial plasma voltage is much greater for the 1.0-torr case. This results in more rapid heating with the result that the emission peak begins earlier in the heating cycle and is much narrower at the lower pressure. In addition, emission intensities are effected by the rate of diffusion of the analyte vapor. The greater peak intensity observed at 4.0 torr is probably the result of a lower diffusion rate.

The use of a $0.64-\mathrm{kG}$ magnetic field results in a ligher plasma voltage and thus greater power dissipation during the early part of the heating cycle. This results in earlier appearance of analyte emission and narrower emission peaks. The greater peak intensity observed at 4.0 torr with the stronger field is probably the result of a reduced rate of radial electron diffusion [13], resulting in more efficient excitation by electron impact.

The continuum background intensity is much greater at the lower pressure for both field strengths. At both pressures, the steady-state background intensity with the 1.24$\mathrm{kG}$ field is about a factor of two lower than with the $0.64-\mathrm{kG}$ field.

\subsection{Hollow cathode}

The hollow cathode furnace configuration operates at significantly lower plasma voltage than the cylindrical post cathode configuration, and no magnetic field was needed for the plasma currents used here. However, some experiments were conducted with the magnetic field present in order to determine its effect on the furnace temperature and plasma voltage.

Voltage vs current plots for the hollow cathode configuration are shown in Fig. 5. Data were collected at Ar pressures of $0.5,1.0,2.0$ and 4.0 torr. These voltage measurements were made after the cathode had achieved a steady-state temperature. For the 1.0-torr case, voltage vs current plots are shown without a magnetic field and with $0.64-$ and $1.24-\mathrm{kG}$ fields. For a pressure of $0.5 \mathrm{torr}$, the plasma became erratic in the presence of the magnetic field. For the 2.0- and 4.0-torr cases, the effect of the magnetic field is much smaller than for the 1.0-torr case.

Note that for all pressures, the voltage increase with increasing current is sub-linear. This is typical for an abnormal glow discharge at high current density values. In addition, the increased contribution of thermionic electrons for the higher temperatures obtained at the larger current values results in a further reduction in the slope of the plots at the higher current values.

The lower voltage of the hollow cathode configuration results in significantly lower power dissipation, and the hollow cathode furnace heats slower than the cylindrical post furnace. Figure 6 shows steady-state surface temperature measurements for the hollow cathode configuration with Ar pressures of 0.5 torr (A), 1.0 torr (B), 2.0 torr (C) and 4.0 torr (D). No magnetic field was used. These temperature measurements were made on the outer surface of the cathode; while most of the positive ion bombardment of the cathode occurs in the cavity. Because of the very small cavity diameter, it was not practical to measure the inner surface temperature. Thus, the values plotted in Fig. 6 may underestimate the effective temperature of the inner furnace wall where the solution residue sample would be located.

The furnace temperatures are significantly lower than the values obtained for the cylindrical post cathode. This is consistent with the larger surface area of the hollow cathode configuration (see Fig. 2). The effect of Ar pressure is greater than for the 


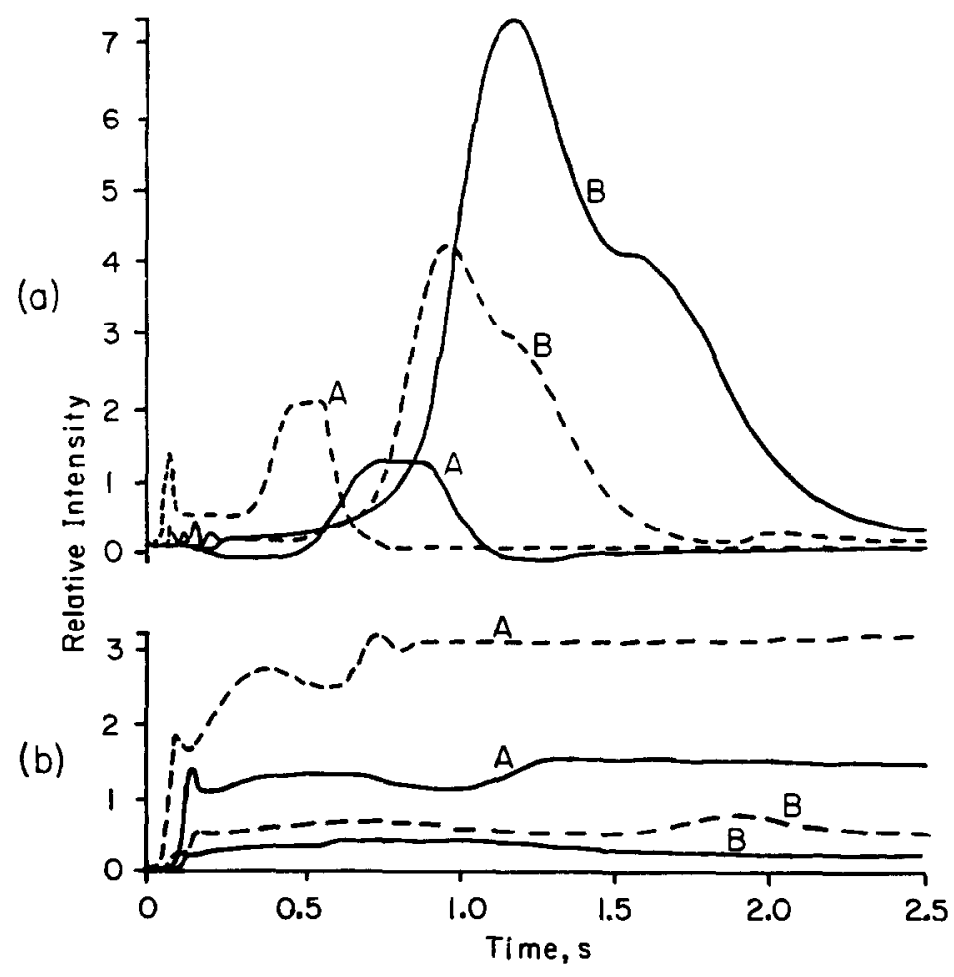

Fig. 4. Emission intensity vs time waveforms for the Mn 403.08-nm neutral-atom line (a) and continuum background (b) for 5.0-ng Mn samples using the cylindrical post furnace. The traces labeled A and B were obtained at Ar pressures of 1.0 and 4.0 torr, respectively. Solid-line traces are for the case with a 1.24-kG magnetic field applied parallel to the electrode axis. Broken-line traces are for the case with a $0.64-\mathrm{kG}$ field.

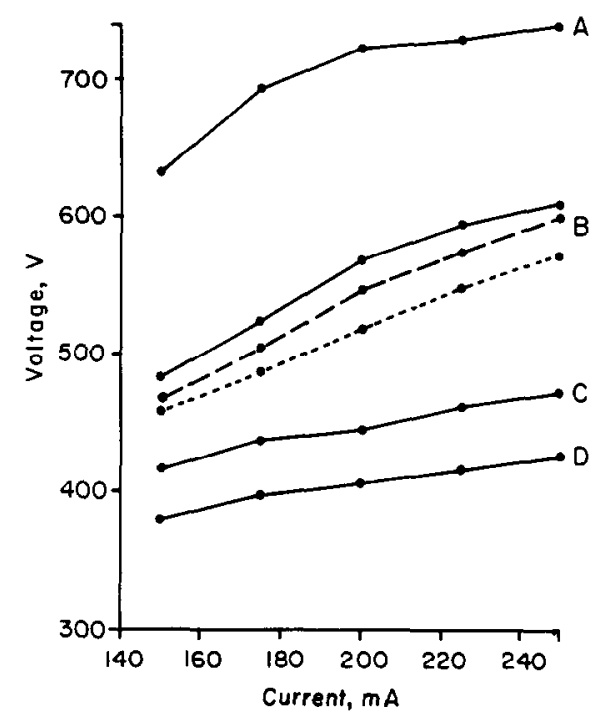

Fig. 5. Plasma voltage vs current waveforms for the hollow cathode furnace for Ar pressures of 0.5 torr (A), 1.0 torr (B), 2.0 torr (C) and 4.00 torr (D). For the 1.0 -torr plots, the solid line is for the case with no magnetic field, while the broken line and dotted line are for the cases with 0.64 - and $1.24-\mathrm{kG}$ fields, respectively. 


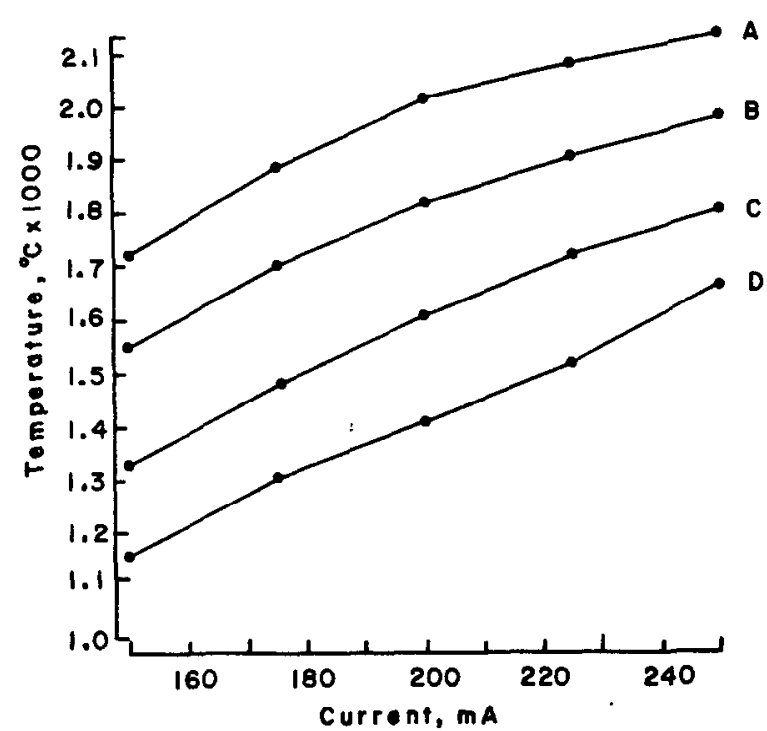

Fig. 6. Plots of furnace temperature vs plasma current for the hollow cathode furnace with Ar pressures of 0.50 torr (A), 1.0 torr (B), 2.0 torr (C) and 4.0 torr (D). No magnetic field was used.

cylindrical post geometry. In addition, the furnace could be operated at pressures as low as 0.5 torr. This is the result of the much lower plasma voltage with the hollow cathode configuration.

Figure 7 shows the effect of an applied magnetic field on the hollow cathode furnace temperature. For the lower pressure, the effect of the magnetic field is significant with lower temperatures obtained for the stronger magnetic field. The effect of the magnetic field is somewhat greater for the lower plasma currents. At a current of $150 \mathrm{~mA}$, the temperature is about $280^{\circ} \mathrm{C}$ lower with the $1.24-\mathrm{kG}$ field relative to the case with no magnetic field. When the Ar presssure is increased to 4.0 torr, the effect of the magnetic field is significantly reduced. In addition, the greatest effect of the magnetic field is observed for the largest plasma current values.

The large increase in plasma voltage and thus power dissipation at the lower pressures probably accounts for the higher temperatures observed at the lower pressures in Figs

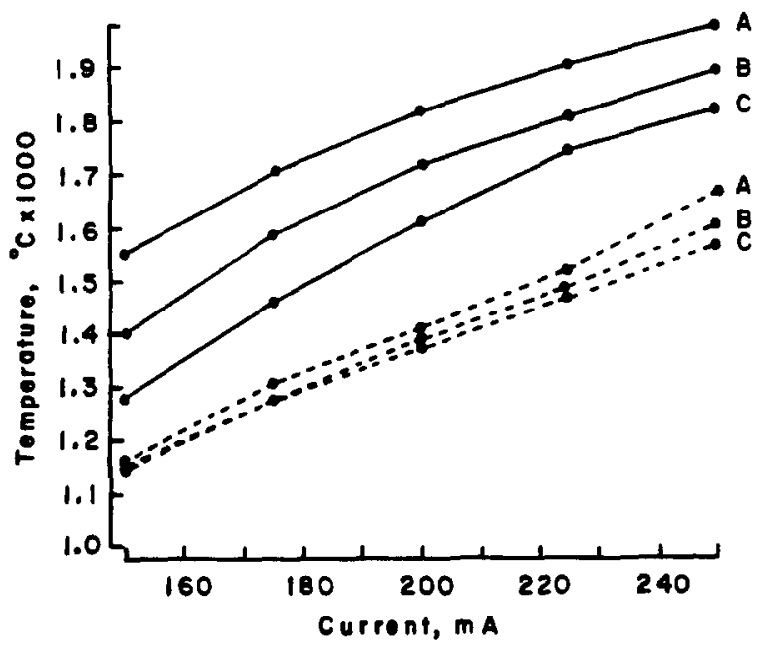

Fig. 7. Plots of furnace temperature vs plasma current for the hollow cathode furnace with Ar pressures of 1.0 torr (solid-line plots) and 4.0 torr (dotted-line plots). For plots labeled A, no magnetic field was used; for plots labeled $B$ and $C, 0.64$ - and 1.24-kG magnetic fields were applied parallel to the cathode axis. 
6 and 7. The effect of the magnetic field in reducing furnace temperature also may be explained by the effect of the field on plasma voltage. The presence of the magnetic field parallel to the hollow cathode axis results in a reduction in the rate of radial electron diffusion $[13,14]$. The resulting increased electron density near the cathode surface increases the efficiency of Ar ionization with the result that the plasma can be maintained at a lower voltage $[15,16]$. In the case of the cylindrical post cathode furnace, the plasma voltage is much greater, particularly when the cathode is too cold for significant thermionic electron emission, and the magnetic field was necessary to reduce the voltage to more tractable values.

Figures 8 and 9 show the effects of plasma current on the emission intensity vs time profiles for the Mn 403.1-nm neutral-atom line and continuum background at the same wavelength for Ar pressures of 1.0 and 4.0 torr. In each figure, emission profiles are shown for three values of plasma current. For the Mn traces, 0.5-ng Mn samples were deposited in the hollow cathode as $5 \mu \mathrm{l}$ of $0.1 \mu \mathrm{g} / \mathrm{ml} \mathrm{Mn}\left(\mathrm{NO}_{3}\right)_{2}$ solution. A distilledwater blank was used for the background traces. Note that the emission intensities are significantly greater with the hollow cathode configuration, and the Mn concentration is a factor of ten lower than for the cylindrical post cathode (see Fig. 4).

The slower heating rate of the hollow cathode configuration is reflected in the significantly broader $\mathrm{Mn}$ emission peaks. Note that for the 4-torr cases, the main emission peak is over within about $2.0 \mathrm{~s}$ for the cylindrical post furnace; while they have barely begun after $2.0 \mathrm{~s}$ for the hollow cathode case.

For a plasma current of $175 \mathrm{~mA}$, the heating is insufficient at 1.0 torr, and only a small emission peak is observed about $4 \mathrm{~s}$ after the start of the heating cyclc. The much lower plasma voltage at 4.0-torr results in very slow heating, and the peak emission intensity is very low and delayed until 8-10 s after the start of the cycle. As the plasma current is increased, the Mn emission peaks are shifted to earlier time and become significantly narrower. These trends are more dramatic for the 4.0-torr case. However, even at $250 \mathrm{~mA}$, the $\mathrm{Mn}$ emission intensity has not fallen to zero after $10 \mathrm{~s}$ of furnace operation.

The continuum background intensity is significantly greater at the lower pressure. This is consistent with the greater plasma voltage and thus greater power dissipation at 1.0 torr. Note that for the 1.0-torr case the background profiles show considerable structure with the intensities increasing rapidly early in the heating cycle, becoming nearly constant during the interval of peak Mn emission and then increasing again as the $\mathrm{Mn}$ signal decays. For the 4.0-torr case, the continuum intensity shows less

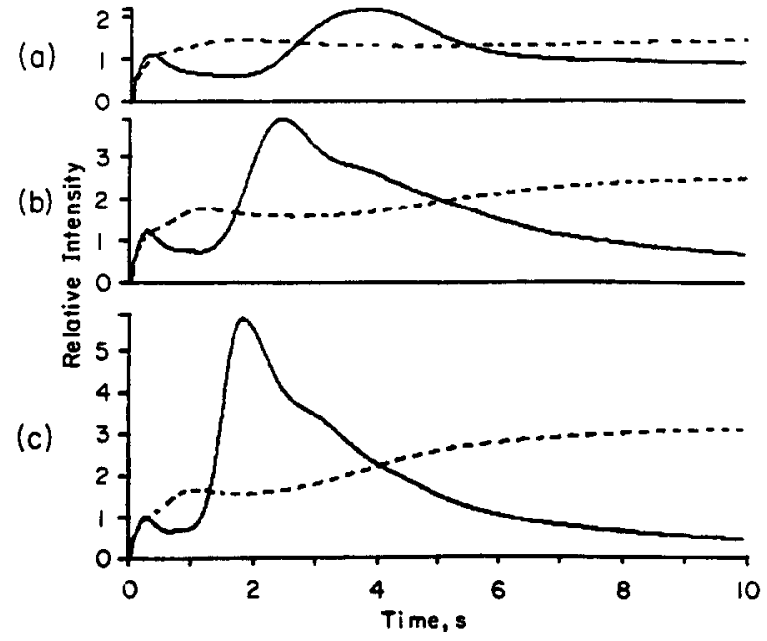

Fig. 8. Emission intensity vs time waveforms for the Mn 403.08-nm neutral-atom line (solidline traces), and continuum background (broken-line traces) for 0.5-ng Mn samples using the hollow cathode furnace with an Ar pressure of 1.0 torr. (a) $175 \mathrm{~mA}$; (b) $225 \mathrm{~mA}$; (c) $250 \mathrm{~mA}$. 
(a)

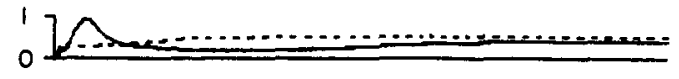

(b)

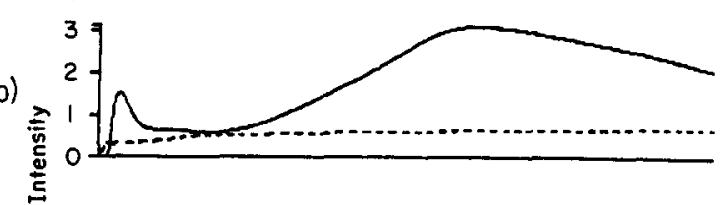

(c)

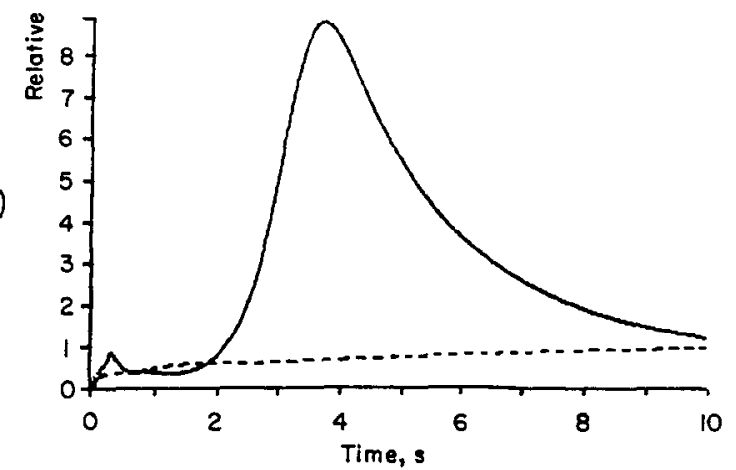

Fig. 9. Emission intensity vs time waveforms for the Mn 403.08-nm neutral-atom line (solidline traces), and continuum background (broken-line traces) for 0.5-ng Mn samples using the hollow cathode furnace with an Ar pressure of 4.0 torr. (a) $175 \mathrm{~mA}$; (b) $225 \mathrm{~mA}$; (c) $250 \mathrm{~mA}$.

structure, increasing gradually over the duration of the heating cycle. This is most apparent for the 250-mA plasma current.

The Mn emission intensity as well as the line-to-background intensity ratio is greatest at the highest current and the highest pressure used in this study. For pressures greater than about 4.0 torr, arcing and plasma instability were significant problems. This problem is amplified by the high cathode current density values needed for efficient furnace heating.

\section{Conclusions}

The use of higher pressures results in greater emission intensities for both furnace configurations. This may be the result of greater sample vapor residence time in the excitation region of the plasma. Higher pressure as well as the use of an axial magnetic field also may result in a more spatially localized space-charge sheath near the furnace surface. This, in turn, may increase the efficiency of sample excitation by electron impact. For the hollow cathode furnace, other processes result in a narrowing of the space-charge sheath [17], and thus the presence of the magnetic field produces a smaller effect.

The advantages of the hollow cathode configuration over the cylindrical post configuration are clearly seen in the nearly order-of-magnitude greater $\mathrm{Mn}$ line intensity and line-to-background intensity ratios. This is observed despite much slower heating and significantly lower peak furnace temperature. This is probably the result of longer vapor residence time and more efficient optical coupling of the emitting zone to the spectrometer. Comparing the 4.0 torr emission profiles at 225 and $250 \mathrm{~mA}$ in Fig. 9 suggests that increasing further the plasma current could significantly increase both analyte intensities and line-to-background ratios. Note that for the 4.0-torr, 250-mA case in Fig. 9, only about $100 \mathrm{~W}$ is dissipated by the hollow cathode furnace.

The preliminary results reported here clearly illustrate the utility of ion bombardment from a low-pressure glow discharge plasma as a means of heating a graphite furnace to temperatures suitable for atomic spectroscopy. The thermal emission of electrons from the hot cathode results in some temperature regulation of the furnace and also may impose an upper limit on furnace temperature. While the resulting plasma appears 
to be a useful excitation source, further characterization is required, particularly regarding the role of thermionic electron emission from the cathode surface.

\section{REFERENCES}

[1] H. Falk, E. Hoftman and Ch. Ludke, Spectrochim. Acta 36B, 767 (1981).

[2] H. Falk, E. Hoffman and Ch. Ludke, Fresenius' Z. Anal. Chim. 307, 362 (1981).

[3] H. Falk, E. Hoffman, Ch. Ludke. J. M. Ottaway and S. K. Giri, Analyst 108, 1459 (1983).

[4] H. Falk, E. Hoffman and Ch. Ludke, Spectrochim. Acta 39B, 283 (1984).

[5] H. Falk, E. Hoffman and Ch. Ludke, Prog. Anal. Spectrosc. 11, 417 (1988).

[6] J. M. Harnly, D. L. Styris and N. E. Ballou. J. Anal. At. Spectrom. 5, 139 (1990).

[7] N. E. Ballou, D. L. Styris and J. M. Harnly, J. Anal. At. Spectrom. 3, 1141 (1988)

[8] S. Tanguay and R. Sacks, Appl. Spectrosc. 43, 918 (1989).

[9] A. von Engel, Ionized Gases. Clarendon Press, Oxford (1965).

[10] D. A. Wright, Proc. Inst. Elec. Engrs, 100, 125 (1953).

[11] S. M. Rossnagel and H. R. Kaufman, J. Vac. Sci. Technol. A4, 1822 (1986).

[12] S. M. Rossnagel, J. Vac. Sci. Technol. A6, 19 (1988).

[13] R. Simonneau and R. Sacks, Appl. Spectrosc. 43, 141 (1989).

[14] R. N. Franklin, Plasma Phenomena in Gas Discharges. Clarendon Press, Oxford (1976).

[15] S. Tanguay and R. Sacks, Appl. Spectrosc. 42, 576 (1988).

[16] J. A. Thornton and A. S. Penfold, Cylindrical magnetron sputtering, in Thin Film Processes, Eds J. L. Vossen and J. J. Cuomo. Academic Press, New York (1978).

[17] M. E. Pillow, Spectrochim. Acta 36B, 821 (1981). 